\title{
Treatment of Acute Coronary Syndrome by Telemedically Supported Paramedics Compared With Physician-Based Treatment: A Prospective, Interventional, Multicenter Trial
}

Jörg C Brokmann ${ }^{1}$, MD; Clemens Conrad ${ }^{2}$, MD; Rolf Rossaint ${ }^{2}$, MD; Sebastian Bergrath ${ }^{2}$, MD; Stefan K Beckers ${ }^{2}$, MD; Miriam Tamm³, Dipl-Math; Michael Czaplik², MD; Frederik Hirsch², MD

\footnotetext{
${ }^{1}$ Emergency Department, Rheinisch-Westfälische Technische Hochschule, University Hospital RWTH Aachen, Aachen, Germany

${ }^{2}$ Department of Anaesthesiology, Rheinisch-Westfälische Technische Hochschule, University Hospital RWTH Aachen, Aachen, Germany

${ }^{3}$ Department of Medical Statistics, Rheinisch-Westfälische Technische Hochschule, University Hospital RWTH Aachen, Aachen, Germany
}

\section{Corresponding Author:}

Frederik Hirsch, MD

Department of Anaesthesiology

Rheinisch-Westfälische Technische Hochschule

University Hospital RWTH Aachen

Pauwelsstrasse 30

Aachen, 52074

Germany

Phone: 49024188179

Fax: 49024182406

Email: fhirsch@ukaachen.de

\section{Abstract}

Background: Prehospital treatment of acute coronary syndrome (ACS) in German emergency medical services (EMSs) is reserved for EMS physicians due to legal issues.

Objective: The objective of this prospective, interventional, multicenter trial was to evaluate the quality of telemedically-delegated therapy and the possible complications in patients with ACS.

Methods: After approval by the ethics committee and trial registration, a one-year study phase was started in August 2012 with 5 ambulances, telemedically equipped and staffed with paramedics, in 4 German EMS districts. The paramedics could contact an EMS-physician-staffed telemedicine center. After initiation of an audio connection, real-time data transmission was automatically established. If required, 12-lead electrocardiogram (ECG) and still pictures could be sent. Video was streamed from inside each ambulance. All drugs, including opioids, were delegated to the paramedics based on standardized, predefined algorithms. To compare telemedically-delegated medication and treatment in ACS cases with regular EMS missions, a matched pair analysis with historical controls was performed.

Results: Teleconsultation was performed on 150 patients having a cardiovascular emergency. In 39 cases, teleconsultation was started due to suspected ACS. No case had a medical complication. Correct handling of 12-lead ECG was performed equally between the groups (study group, $\mathrm{n}=38$ vs control group, $\mathrm{n}=39, P>.99$ ). There were no differences in correct handling of intravenous administration of acetylsalicylic acid, heparin, or morphine between both the groups (study group vs control group): acetylsalicylic acid, $\mathrm{n}=31$ vs $\mathrm{n}=33, P=.73$; unfractionated heparin, $\mathrm{n}=34$ vs $\mathrm{n}=33, P>.99$; morphine, $\mathrm{n}=29$ vs $\mathrm{n}=27, P=.50$. The correct handling of oxygen administration was significantly higher in the study group ( $\mathrm{n}=29 \mathrm{vs} \mathrm{n}=18, P=.007)$.

Conclusions: Telemedical delegation of guideline conform medication and therapy by paramedics in patients with ACS and was found to be feasible and safe. The quality of guideline-adherent therapy was not significantly different in both the groups except for the correct administration of oxygen, which was significantly higher in the study group.

Trial Registration: Clinicaltrials.gov NCT01644006; http://clinicaltrials.gov/ct2/show/NCT01644006 (Archived by WebCite at http://www.webcitation.org/6mPam3eDy).

(J Med Internet Res 2016;18(12):e314) doi: 10.2196/jmir.6358 


\section{KEYWORDS}

acute coronary syndrome; prehospital emergency care; telemedicine; telehealth; myocardial infarction

\section{Introduction}

The in-hospital mortality rate of ST Segment Elevation Myocardial Infarction (STEMI) could be significantly reduced by the modern reperfusion therapy and improved secondary prophylaxis. However, the overall mortality remains unchanged because two-thirds of deaths occur during the prehospital phase-usually due to lethal arrhythmias that are triggered by ischemic events [1]. Therefore, an appropriate therapy must be initiated in the early prehospital phase, and the time of admission must be as small as possible to improve survival. This is also important in non-STEMI-acute coronary syndrome (NSTE-ACS) as the incidence of NSTE-ACS rises further [2,3].

Telemedical support for diagnosis and therapy in acute coronary syndromes (ACSs) has been established for quite some time in emergency medical services (EMS). Several telemetry projects have demonstrated feasibility [4-7] in the transmission of a 12-lead electrogram (ECG) or its images to a cardiologist. The cardiologist receiver can then consecutively assess the potential to improve survival and outcome in STEMI [7]. It even has been shown that an artificial neural network could predict STEMI and the need of acute percutaneous coronary intervention (PCI) in ambulance ECGs [8]. However, all of these studies assessed the impact of 12-lead ECG transmission on in-hospital parameters and outcome in STEMI patients. Evidence regarding telemedical concepts in non-STEMI ACS patients is mostly lacking.

Germany uses a two-tiered EMS system with paramedic-staffed ambulances and additional EMS with physician response units. Like some other European countries, only an EMS physician can administer the medication required in ACS. Except for nitrates, paramedics cannot dispense drugs. In cases where primary dispatch was merely a paramedic-staffed ambulance and the EMS physician was notified later, the time to arrival and thus the administration of medication may be unacceptably long. This effect is exacerbated in more rural areas and by an occasional lack of physician-staffed EMS $[9,10]$.

Against this background, a mobile telemedicine system was developed during a first research project from 2007 to 2010. Two comprehensive simulation studies demonstrated that real-time telemedical support by experienced remote EMS physicians leads to improved quality of care in STEMI. Even telemedically supported paramedics were able to handle emergency care on their own with a comparable performance compared with on-scene physicians [11,12]. The main findings during the project, preceding to the one described here, were the feasibility of prehospital teleconsultation in general and the improvement of data transmission in acute stroke [13,14]. This succeeding research project allowed further technical and organizational development. The telemedical equipment was made more practicable and paramedic-staffed ambulances were equipped with this system. Thus, the concept allowed medications to be given by paramedics in ACS when supervised by an experienced EMS physician-the so-called tele-EMS physician. In this prospective, interventional trial, we assessed the quality of prehospital emergency care in patients suffering from ACS (measured by adherence to international guidelines) when using a telemedically supported EMS. Using matched pairs, we compared our findings with a comparable historical control group treated by our regular physician-based EMS.

\section{Methods}

This prospective, interventional, multicenter trial was conducted from August 1, 2012 to July 31, 2013 within the research project "TemRas" (telemedical rescue assistance system).

\section{Study Setting}

In 4 different EMS districts in Germany, 5 paramedic-staffed ambulances were stepwise equipped with a multifunctional telemedicine system. Three of the 4 EMS districts were rural and one was urban (Table 1). As described elsewhere, all participating EMS physicians in the function of a "tele-EMS physician" were trained prior to the start of the intervention phase [15]. All participating tele-EMS physicians had a minimum experience of 3 years in anesthesia and critical care as well as broad experience as an EMS physician. The paramedics involved $(\mathrm{N}=178)$ ran through a standardized eight-hour training program before the project started. The goal was to learn the use of the technical system, the medical concept of teleconsultation including indications for teleconsultation (eg, ACS), and communication skills [15]. 
Table 1. Demographics and structure of the participating emergency medical service (EMS) districts.

\begin{tabular}{|c|c|c|c|c|}
\hline Demographics & $\begin{array}{l}\text { Aachen } \\
\text { (urban) }\end{array}$ & $\begin{array}{l}\text { Heinsberg } \\
\text { (rural) }\end{array}$ & $\begin{array}{l}\text { Dueren } \\
\text { (rural) }\end{array}$ & $\begin{array}{l}\text { Euskirchen } \\
\text { (rural) }\end{array}$ \\
\hline Population & 248,137 & 256,546 & 267,712 & 190,591 \\
\hline Area $\left(\mathrm{km}^{2}\right)$ & 160.8 & 628.0 & 941.4 & 1248.7 \\
\hline \multicolumn{5}{|l|}{ Ambulances } \\
\hline 24-h Ambulances (telemedically equipped ${ }^{\mathrm{a}}$ ) & $6(2)$ & $7(1)$ & $11(1)$ & $9(1)$ \\
\hline Daytime ambulances & 2 & 3 & 2 & 2 \\
\hline EMS $^{\mathrm{b}}$ physician units & $2+1^{\mathrm{c}}$ & 4 & $4+1^{d}$ & 3 \\
\hline Ambulance emergency missions/year & 22,984 & 14,346 & 20,302 & 15,108 \\
\hline EMS physician missions/year & 7898 & 7786 & 9057 & 5317 \\
\hline Hospitals & 4 & 4 & 5 & 3 \\
\hline Stroke units & 1 & 1 & 1 & 1 \\
\hline Level 1 trauma center & 1 & - & - & - \\
\hline 24-h Cardiac catheterization laboratory & 1 & 1 & 1 & 1 \\
\hline Number of cases (n) & 18 & 6 & 3 & 12 \\
\hline
\end{tabular}

atelemedically equipped for 24 hours, operation of teleconsultation center: 7:30 am-4:30 pm.

${ }^{b}$ EMS: emergency medical service.

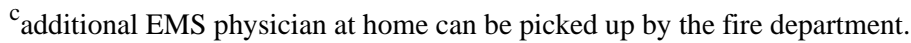

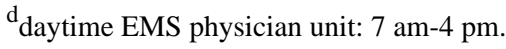

\section{Trial Registration and Ethical Issues}

The trial was registered before the intervention phase (clinicaltrials.gov NCT01644006). The study was approved by the local ethics committee (University Hospital Rheinisch-Westfälische Technische Hochschule Aachen, Germany, registration number EK 191/11). The written informed consent was waived by the ethics committee due to the emergency setting; however, all alert patients had to give verbal approval to data and video transmission before teleconsultation. Data from the preintervention phase was analyzed retrospectively via prospectively planned outcomes of the intervention phase. Multimedia Appendix 1 shows the trial protocol.

\section{Technical System}

An MRx monitor-defibrillator unit (Philips Healthcare, Andover, MA, USA) connected to a portable data transmission unit (peeqBox, P3 communications, Aachen, Germany) was used by the paramedics to establish parallelized and encrypted data and audio connection via different mobile networks. The audio channel was accomplished with 2 headsets (Voyager Pro HD, Plantronics, Santa Cruz, CA, USA) connected via Bluetooth to the mobile transmission unit. Real-time vital data transmission (numerical values and curves, Einthoven leads I-III continuously) occurred automatically after initiation of a teleconsultation. If required, 12-lead ECGs could be transmitted. Still pictures could be sent using a Bluetooth connection between a mobile phone (HTC Sensation XE, High Tech Computer Corporation, Taoyuan, Taiwan) and the data transmission unit. The tele-EMS physician could start a video streaming from a ceiling camera of the ambulance when the patient was inside in the ambulance. On the telemedical workstation, real-time vital data was displayed using the IntelliVue Information Centre (Philips Healthcare, Boeblingen, Germany), and 12-lead-ECGs were displayed on the HeartStart Telemedicine Viewer (Philips Healthcare, Andover, MA, USA). All other software components in the telemedicine center were specifically developed as part of the research project. In cases of ACS, an established algorithm could be displayed on the workstation to support the tele-EMS physician in guideline-adherent therapy recommendations including the steps needed to diagnose and dose medication as recommended by national and international guidelines. A detailed technical description of the telemedicine system was already described elsewhere [16].

\section{Interventions}

The decision to initiate a teleconsultation was solely made by the on-scene paramedics. All participating EMS districts ran their own dispatch centers. Local protocols for ambulance and EMS physician units' alarm were not changed before or within the project period. The telemedicine system was used in addition to the regular EMS system. If the EMS dispatch centers suspected ACS, then an EMS physician response unit was sent in accordance to the special provisions of law for paramedic-staffed ambulance. Therefore, only cases where the initial notification was different or cases that bridged the arrival of an EMS physician unit could be managed with telemedical care. The additional telemedicine service was available during workdays from 7:30 am to $4: 30 \mathrm{pm}$ during the first 4 months of the intervention phase and from 7:30 am to 8:00 pm for the rest of the trial phase due to restricted funding. No teleconsultation system existed during the preintervention period. 


\section{Outcomes and Data Sources}

The following data sources were used: the teleconsultation protocols, paper-based EMS protocols of the participating EMS stations, and data of the local EMS dispatch centers. The quality of prehospital care of ACS based on national and international published guidelines [17-21] for non-STEMI-ACS and STEMI was analyzed as the primary outcome measure. Guideline adherence was measured by correct handling of the following measures: 12-lead ECG completed; administration of acetylsalicylic acid $\geq 250 \mathrm{mg}$ iv, independent of long-term therapy of the patient; administration of heparin $70 \mathrm{IU} / \mathrm{kg}$ iv, maximum $5000 \mathrm{IU}$, but waiver in the presence of effective anticoagulation in patient's long-term therapy; administration of morphine if numerical rating scale (NRS) $\geq 3$ (repeatedly $3-5 \mathrm{mg}$ iv until NRS $\leq 3$ ); and administration of oxygen if oxygen saturation $\leq 95 \%$. Treatment was not carried out in cases of any allergies or contraindications. If so, this was measured as correct handling.

A standardized report for any potential problems or adverse events was completed daily by the tele-EMS physician in charge. The adverse events were defined as follows: respiratory or circulatory insufficiency due to administered medications with a need for intervention or allergic reaction due to administered medications. Multimedia Appendix 2 shows the original dataset for further statistical evaluation.

\section{Sample Size}

The trial had a restricted funding period of one year. Because this was the first study of prehospital telemedically-delegated diagnosis and therapy including medication in patients with ACS in this setting, a power analysis and formal sample size calculation could not be performed before this pilot study. A possible sample size was estimated by an expert group and documented in the trial registration. The anticipated enrolment of the sample size was $\mathrm{N}=180$.

\section{Inclusion and Exclusion Criteria}

Patients were included if ACS was diagnosed, verbal consent for teleconsultation was obtained, and were aged $\geq 18$ years. Patients who refused consent to teleconsultation or had no suspected ACS were excluded. Figure 1 shows the study flowchart.

\section{Matched Pairs}

The matched pairs were searched from a historical preintervention period (April 1, 2011 to March 31, 2012) that was one consecutive year prior to the first training lesson. Matching was done using predefined criteria (Table 2) and the nearest-neighbour matching method for all cases that fulfilled the matching criteria.

Table 2. Matching criteria.

\begin{tabular}{ll}
\hline Matching criteria & Matching categories \\
\hline EMS $^{\text {a district }}$ & Study group cases and control group cases had to be from same district \\
Date of emergency mission & $\begin{array}{l}\text { During the historical control period, the same month and calendar day was used as starting point for the backward and } \\
\text { forward search; the first case that fulfilled the matching criteria listed below was included }\end{array}$ \\
Patient's age & Same age \pm 10 years \\
Sex & Female \\
Male & Chest pain (typically) \\
Nymptoms & No chest pain (atypically), but other symptoms (ie, nausea, vomiting) \\
& No symptoms
\end{tabular}

${ }^{a}$ EMS: emergency medical service. 
Figure 1. Study flowchart.

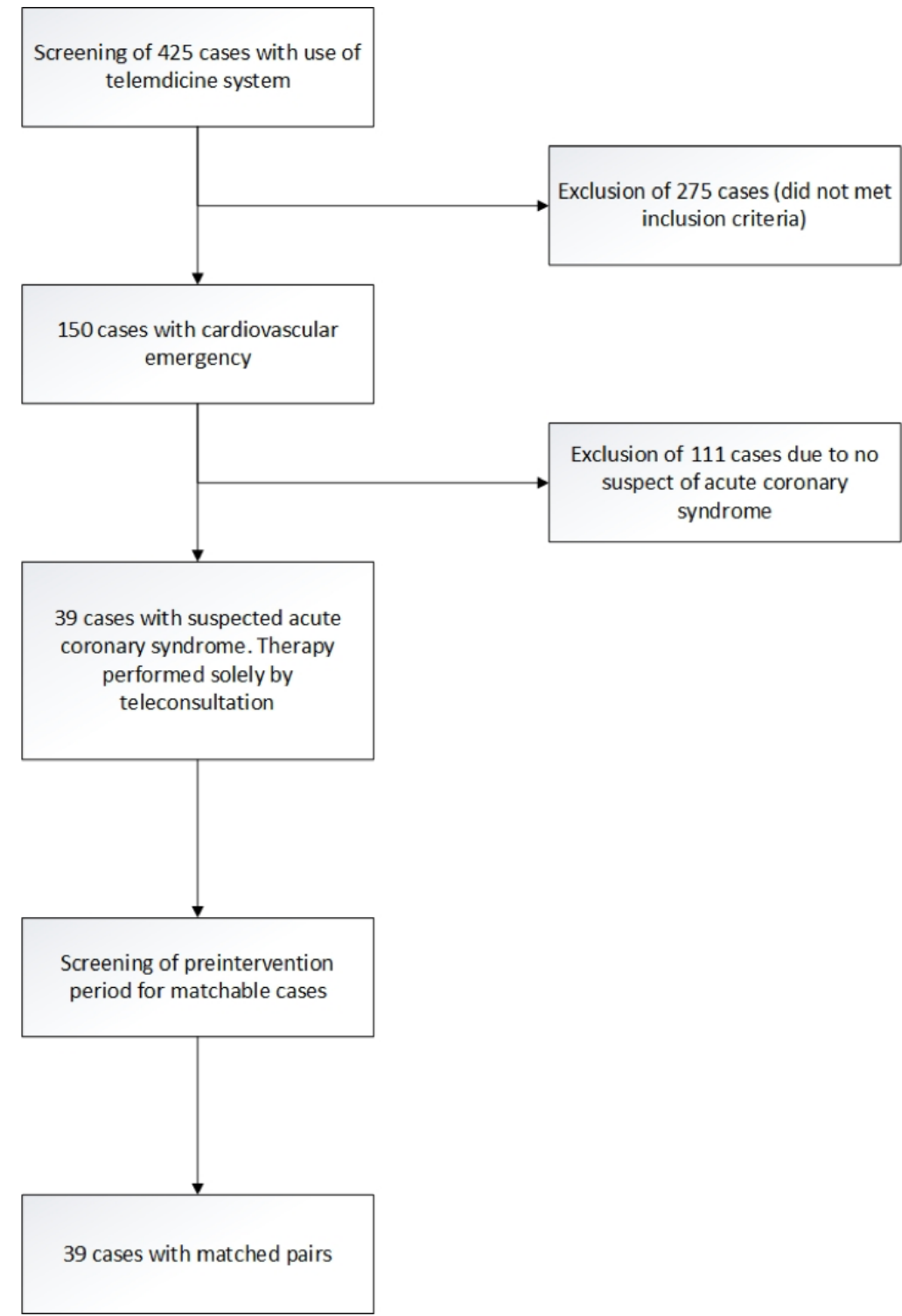

\section{Statistical Methods}

Categorical parameters were compared using 4 field contingency tables with McNemar's Test. All statistical analyses were performed using SAS 9.4 (SAS Institute Inc). $P$ values $<.05$ were considered to be significant. Multimedia Appendix 3 shows the statistical evaluation.

\section{Results}

Overall, 425 emergency teleconsultation cases were performed with 150 patients having a cardiovascular emergency. The demographic data (Multimedia Appendix 4) was spread equally between the study group and the control group. In each group, 14 patients were female $(36 \%, 14 / 39)$, the rest were male. In 39 cases, teleconsultation was started due to suspected ACS. In

the described cases, no adverse events were detected. Correct handling of 12-lead ECG was performed equally between the groups (study group, $\mathrm{n}=38$ vs control group, $\mathrm{n}=39 ; P>.99$ ). There was no significant difference in correct handling of intravenous administration of acetylsalicylic acid $(n=38)$, heparin $(n=38)$, or morphine $(n=29)$ between both groups (study group vs control group): acetylsalicylic acid, $n=31$ vs $n=33, P=.73$; unfractionated heparin, $\mathrm{n}=34$ vs $\mathrm{n}=33, P>.99$; and morphine, $\mathrm{n}=29$ vs $\mathrm{n}=27 \quad P=.50$. The correct handling of oxygen differed significantly between the groups $(n=34)$. In the study group it was performed correctly for $n=29$ and for $n=18$ in the control group $(P=.007)$. Multimedia Appendix 5 gives a detailed view of the correct or incorrect handling of each measure in each of the participating districts. Table 3 summarizes the above mentioned results. 
Table 3. Summary of results of correct handling of measures.

\begin{tabular}{|c|c|c|c|c|}
\hline Measures & $\mathrm{n}$ & Study group & Control group & $P$ value \\
\hline 12-lead ECG ${ }^{\mathrm{a}}$ & 39 & 38 & 39 & $>.99$ \\
\hline Acetylsalicylic acid & 38 & 31 & 33 & .73 \\
\hline Heparin & 38 & 34 & 33 & $>.99$ \\
\hline Morphine & 29 & 29 & 27 & .50 \\
\hline Oxygen & 34 & 29 & 18 & .007 \\
\hline
\end{tabular}

${ }^{a}$ ECG: electrocardiogram.

\section{Discussion}

\section{Principal Findings}

To the best of our knowledge, this is the first study providing prehospital diagnosis and emergency care for ACS that was completely delivered by telemedically supported paramedics. The telemedical concept is feasible, and guideline adherence was at least comparable with on-scene physician care with improved guideline adherence regarding oxygen administration. The feasibility and improvements on outcome by wireless transmission of ECGs to specialists have already been shown in STEMI $[4,5,7]$. As demonstrated here, both groups handled the administration of the 12-lead ECG correctly. Due to legal restrictions in Germany, the full administration of required medication in patients with ACS administered by paramedics is completely new to the field of prehospital care. The required medication for each patient in the study group was delegated by the tele-EMS physician. Between groups, there were no significant differences in the correct handling of intravenous administration of acetylsalicylic acid, heparin, or morphine. It was already shown that telemedically supported administration of analgesics by paramedics is feasible and secure [22]. Regarding the pain therapy in ACS described here, both groups could improve the correct handling of morphine. We noted that morphine should be administered when NRS was $\geq 3$ (repeatedly 3-5 mg iv). Eventually, morphine was given in both groups even if NRS was not $\geq 3$ or pain reduction was not achieved in a sufficient extent.

In the study group, the oxygen administration was performed significantly more correctly than in the control group. This may be because the tele-EMS physician had a predefined algorithm for correct treatment of ACS at any time in digital form that was integrated in the documentation system. As this algorithm stated, oxygen had to be applied when the blood saturation was $\leq 95 \%$. We assume that oxygen was applied in the control group all too often, even if the oxygen saturation was above $95 \%$. Therefore, the differences are explained by improved guideline adherence.

Neither the telemedicine group nor the control group had any medical complication. Due to the small sample size, a definitive statement regarding the safety of complete prehospital ACS therapy by paramedics and tele-EMS physicians cannot be given.
However, these findings support the thesis that telemedically supported prehospital diagnosis and therapy on the whole is a safe procedure, although the process was completely new for the participating physicians as well as for the paramedics. In comparison with ground- or helicopter-based EMS physician operations [23], this concept allows for a spatially unrestricted emergency concept with fewer resources. At this point it has to be mentioned that, to our knowledge, most studies with 12-lead-ECG transmission represent rather small sample sizes. Therefore, a large randomized controlled trial is needed.

No case had major technical problems. Although the technical performance of the telemedicine system has not yet been evaluated in detail, no consultation had to be terminated due to technical problems.

\section{Limitations}

This pilot study had no formal sample size calculation and power analysis. Therefore, all results have to be interpreted against this background. This was the first pilot study to evaluate the concept of telemedically-assisted prehospital therapy in ACS to allow safe, effective, and guideline-adherent therapy. On the basis of these pilot results, a confirmatory trial with a calculated sample size would be meaningful. However, it cannot be definitely stated that telemedically assisted treatment is as effective as treatment with an on-site EMS physician. Due to medical privacy policy in Germany, we could not obtain in-hospital follow-up data and therefore could not highlight outcome parameters such as in-hospital time intervals in STEMI (contact to balloon time, arrival to balloon time), the rate of secondary transfer for PCI (rate of secondary transfer to a different facility for PCI), or laboratory analysis like troponin-levels.

\section{Conclusions}

Prehospital diagnosis and treatment of ACS that was carried out by paramedics supported by tele-EMS physicians seems to be as safe and effective as the conventional procedure, assuming a holistic telemedical support system is used, as shown here. In fact, the supply and the adherence to national and international guidelines was better and not worse than in the conventional EMS physician-based service. Further trials with larger patient numbers and a randomized allocation are needed to confirm the findings of this pilot study. 


\section{Acknowledgments}

The study was conducted within the joint research project "TemRas," funded by the European Union and the Ministry of Innovation, Science and Research of North Rhine Westphalia, Germany (MIWF), Project-No: PtJ-Az. 0909im002b, Funding code 005-1003-0034. Philips Healthcare (Hamburg, Germany) and P3 communications (Aachen, Germany) contributed their own financial resources. The funders had no role in the study design, data collection and analysis, decision to publish, or preparation of the manuscript. No author had financial relationships with or other nonfinancial dependencies on the funding sponsors.

The authors thank their colleagues from the Institute of Information Management in Mechanical Engineering (IMA), Centre for Learning and Knowledge Management (ZLW), Associate Institute for Management Cybernetics eV (IfU), ZLW/IMA, and the P3 communications who provided insight and expertise that greatly assisted the research, although they may not agree with all of the interpretations and conclusions of this article.

\section{Authors' Contributions}

JCB, FH, RR, and SB made substantial contributions to conception and design, acquisition of data, and drafting the manuscript. MC, CC, and SKB participated in study design. CC and FH acquired data. FH, CC, JB, and SB analyzed and interpreted data. MT carried out statistical analysis of data. All authors were involved in drafting the manuscript and revising it critically. All authors read and approved the final manuscript.

\section{Conflicts of Interest}

After the completion of the research project JCB, RR, and MC founded the Docs in Clouds telemedicine service and consulting company.

\section{Multimedia Appendix 1}

Trial protocol.

[PDF File (Adobe PDF File), 881KB-Multimedia Appendix 1]

\section{Multimedia Appendix 2}

Dataset.

[XLSX File (Microsoft Excel File), 15KB-Multimedia Appendix 2]

\section{Multimedia Appendix 3}

Statistical evaluation (raw dataset).

[PDF File (Adobe PDF File), 71KB-Multimedia Appendix 3]

\section{Multimedia Appendix 4}

Demographic data.

[PDF File (Adobe PDF File), 11KB-Multimedia Appendix 4]

\section{Multimedia Appendix 5}

Detail table.

[PDF File (Adobe PDF File), 293KB-Multimedia Appendix 5]

\section{References}

1. Löwel H, Meisinger C, Heier M, Hörmann A, Kuch B, Gostomzyk J, et al. Sex specific trends of sudden cardiac death and acute myocardial infarction: results of the population-based KORA/MONICA-Augsburg register 1985 to 1998. Dtsch Med Wochenschr 2002 Nov 2;127(44):2311-2316. [doi: 10.1055/s-2002-35181] [Medline: 12410433]

2. Goodman SG, Huang W, Yan AT, Budaj A, Kennelly BM, Gore JM, Expanded Global Registry of Acute Coronary Events (GRACE2) Investigators. The expanded Global Registry of Acute Coronary Events: baseline characteristics, management practices, and hospital outcomes of patients with acute coronary syndromes. Am Heart J 2009 Aug;158(2):193-201.e1. [doi: 10.1016/j.ahj.2009.06.003] [Medline: 19619694]

3. Fox KA, Cokkinos DV, Deckers J, Keil U, Maggioni A, Steg G. The ENACT study: a pan-European survey of acute coronary syndromes. European Network for Acute Coronary Treatment. Eur Heart J 2000 Sep;21(17):1440-1449 [FREE Full text] [doi: 10.1053/euhj.2000.2185] [Medline: 10952836] 
4. Adams GL, Campbell PT, Adams JM, Strauss DG, Wall K, Patterson J, et al. Effectiveness of prehospital wireless transmission of electrocardiograms to a cardiologist via hand-held device for patients with acute myocardial infarction (from the Timely Intervention in Myocardial Emergency, NorthEast Experience [TIME-NE]). Am J Cardiol 2006 Nov 1;98(9):1160-1164. [doi: 10.1016/j.amjcard.2006.05.042] [Medline: 17056318]

5. Dhruva VN, Abdelhadi SI, Anis A, Gluckman W, Hom D, Dougan W, et al. ST-Segment analysis using wireless technology in acute myocardial infarction (STAT-MI) trial. J Am Coll Cardiol 2007 Aug 7;50(6):509-513 [FREE Full text] [doi: 10.1016/j.jacc.2007.04.049] [Medline: 17678733]

6. Sejersten M, Sillesen M, Hansen PR, Nielsen SL, Nielsen H, Trautner S, et al. Effect on treatment delay of prehospital teletransmission of 12-lead electrocardiogram to a cardiologist for immediate triage and direct referral of patients with ST-segment elevation acute myocardial infarction to primary percutaneous coronary intervention. Am J Cardiol 2008 Apr 1;101(7):941-946. [doi: 10.1016/j.amjcard.2007.11.038] [Medline: 18359312]

7. Sanchez-Ross M, Oghlakian G, Maher J, Patel B, Mazza V, Hom D, et al. The STAT-MI (ST-Segment analysis using wireless technology in acute myocardial infarction) trial improves outcomes. JACC Cardiovasc Interv 2011 Feb;4(2):222-227 [FREE Full text] [doi: 10.1016/j.jcin.2010.11.007] [Medline: 21349462]

8. Forberg JL, Khoshnood A, Green M, Ohlsson M, Björk J, Jovinge S, et al. An artificial neural network to safely reduce the number of ambulance ECGs transmitted for physician assessment in a system with prehospital detection of ST elevation myocardial infarction. Scand J Trauma Resusc Emerg Med 2012;20:8 [FREE Full text] [doi: 10.1186/1757-7241-20-8] [Medline: 22296816]

9. Reimann B, Maier B, Lott R, Konrad F. Gefaehrdung der Notarztversorgung im laendlichen Gebiet. Notfall \& Rettungsmedizin 2004 May;7(3):200-204. [doi: 10.1007/s10049-004-0656-8]

10. Behrendt H, Schmiedel R. Die aktuellen Leistungen des Rettungsdienstes in der Bundesrepublik Deutschland im zeitlichen Vergleich (Teil II). Notfall \& Rettungsmedizin 2004 Feb 1;7(1):59-70. [doi: 10.1007/s10049-003-0623-9]

11. Skorning M, Bergrath S, Rörtgen D, Beckers SK, Brokmann JC, Gillmann B, et al. Teleconsultation in pre-hospital emergency medical services: real-time telemedical support in a prospective controlled simulation study. Resuscitation 2012 May;83(5):626-632. [doi: 10.1016/j.resuscitation.2011.10.029] [Medline: 22115932]

12. Rörtgen D, Bergrath S, Rossaint R, Beckers SK, Fischermann H, Na I, et al. Comparison of physician staffed emergency teams with paramedic teams assisted by telemedicine--a randomized, controlled simulation study. Resuscitation 2013 Jan;84(1):85-92. [doi: 10.1016/j.resuscitation.2012.06.012] [Medline: 22750663]

13. Bergrath S, Rörtgen D, Rossaint R, Beckers SK, Fischermann H, Brokmann JC, et al. Technical and organisational feasibility of a multifunctional telemedicine system in an emergency medical service - an observational study. J Telemed Telecare 2011;17(7):371-377. [doi: 10.1258/jtt.2011.110203] [Medline: 21933897]

14. Bergrath S, Reich A, Rossaint R, Rörtgen D, Gerber J, Fischermann H, et al. Feasibility of prehospital teleconsultation in acute stroke--a pilot study in clinical routine. PLoS One 2012;7(5):e36796 [FREE Full text] [doi:

10.1371/journal.pone.0036796] [Medline: 22629331]

15. Bergrath S, Czaplik M, Rossaint R, Hirsch F, Beckers SK, Valentin B, et al. Implementation phase of a multicentre prehospital telemedicine system to support paramedics: feasibility and possible limitations. Scand J Trauma Resusc Emerg Med 2013;21:54 [FREE Full text] [doi: 10.1186/1757-7241-21-54] [Medline: 23844941]

16. Czaplik M, Bergrath S, Rossaint R, Thelen S, Brodziak T, Valentin B, et al. Employment of telemedicine in emergency medicine. Clinical requirement analysis, system development and first test results. Methods Inf Med 2014;53(2):99-107. [doi: 10.3414/ME13-01-0022] [Medline: 24477815]

17. Hamm CW. Guidelines: Acute coronary syndrome (ACS). II: Acute coronary syndrome with ST-elevation. Z Kardiol 2004 Apr;93(4):324-341. [doi: 10.1007/s00392-004-0109-x] [Medline: 15085379]

18. Hamm CW. Guidelines: acute coronary syndrome (ACS). 1: ACS without persistent ST segment elevations. Z Kardiol 2004 Jan;93(1):72-90. [doi: 10.1007/s00392-004-1064-2] [Medline: 14740245]

19. Task Force on the management of ST-segment elevation acute myocardial infarction of the European Society of Cardiology (ESC), Steg PG, James SK, Atar D, Badano LP, Blömstrom-Lundqvist C, et al. ESC Guidelines for the management of acute myocardial infarction in patients presenting with ST-segment elevation. Eur Heart J 2012 Oct;33(20):2569-2619 [FREE Full text] [doi: 10.1093/eurheartj/ehs215] [Medline: 22922416]

20. Hamm CW, Bassand JP, Agewall S, Bax J, Boersma E, Bueno H, ESC Committee for Practice Guidelines. ESC Guidelines for the management of acute coronary syndromes in patients presenting without persistent ST-segment elevation: The Task Force for the management of acute coronary syndromes (ACS) in patients presenting without persistent ST-segment elevation of the European Society of Cardiology (ESC). Eur Heart J 2011 Dec;32(23):2999-3054 [FREE Full text] [doi: 10.1093/eurheartj/ehr236] [Medline: 21873419]

21. Antman EM, Anbe DT, Armstrong PW, Bates ER, Green LA, Hand M, American College of Cardiology/American Heart Association Task Force on Practice Guidelines (Writing Committee to Revise the 1999 Guidelines for the Management of Patients With Acute Myocardial Infarction). ACC/AHA guidelines for the management of patients with ST-elevation myocardial infarction--executive summary: a report of the American College of Cardiology/American Heart Association Task Force on Practice Guidelines (Writing Committee to Revise the 1999 Guidelines for the Management of Patients With 
Acute Myocardial Infarction). Circulation 2004 Aug 3;110(5):588-636 [FREE Full text] [doi: 10.1161/01.CIR.0000134791.68010.FA] [Medline: 15289388]

22. Brokmann JC, Rossaint R, Hirsch F, Beckers SK, Czaplik M, Chowanetz M, et al. Analgesia by telemedically supported paramedics compared with physician-administered analgesia: A prospective, interventional, multicentre trial. Eur J Pain 2016 Aug;20(7):1176-1184. [doi: 10.1002/ejp.843] [Medline: 26914284]

23. Schuster M, Pints M, Fiege M. Duration of mission time in prehospital emergency medicine: effects of emergency severity and physicians level of education. Emerg Med J 2010 May;27(5):398-403. [doi: 10.1136/emj.2009.074211] [Medline: 20442179]

\author{
Abbreviations \\ ACS: acute coronary syndrome \\ ECG: electrocardiogram \\ EMS: emergency medical service \\ iv: intravenous \\ IU: international units \\ NRS: numeric rating scale \\ NSTE-ACS: non-STEMI-acute coronary syndrome \\ PCI: percutaneous coronary intervention \\ STEMI: ST elevation myocardial infarction \\ TemRas: Telemedical rescue assistance system
}

Edited by G Eysenbach; submitted 15.07.16; peer-reviewed by S Omboni, N Bashi; comments to author 25.08.16; revised version
received 10.10.16; accepted 15.10.16; published 01.12.16
Please cite as:
Brokmann JC, Conrad C, Rossaint R, Bergrath S, Beckers SK, Tamm M, Czaplik M, Hirsch F
Treatment of Acute Coronary Syndrome by Telemedically Supported Paramedics Compared With Physician-Based Treatment: A
Prospective, Interventional, Multicenter Trial
J Med Internet Res 2016;18(12):e314
URL: $\underline{\text { http://www.jmir.org/2016/12/e314/ }}$
doi: $\underline{10.2196 / j m i r .6358}$
PMID: $\underline{27908843}$

CJörg C Brokmann, Clemens Conrad, Rolf Rossaint, Sebastian Bergrath, Stefan K Beckers, Miriam Tamm, Michael Czaplik, Frederik Hirsch. Originally published in the Journal of Medical Internet Research (http://www.jmir.org), 01.12.2016. This is an open-access article distributed under the terms of the Creative Commons Attribution License (http://creativecommons.org/licenses/by/2.0/), which permits unrestricted use, distribution, and reproduction in any medium, provided the original work, first published in the Journal of Medical Internet Research, is properly cited. The complete bibliographic information, a link to the original publication on http://www.jmir.org/, as well as this copyright and license information must be included. 